\section{Rhino protection in Africa}

SIR-The Commentary by LeaderWilliams and Albon (Nature 336, 533; 1988), on the allocation of resources for conservation of African big game, leads me to enquire whether any consideration has been given to dehorning of wild rhinoceroses.

For the past 30 years a dehorned male rhinoceros has lived in the National Zoological Park in Washington, DC. Dehorned animals may be handicapped in ritual sparring, and the attractiveness of rhinos for tourists might be marginally reduced (although countered by raised awareness of the poaching problem), but elimination of the rationale for rhino slaughter seems to be a simple and inexpensive measure that might save the species.

JOHN BuCK

4505 Saul Road, Kensington, Maryland 20895, USA

SIR-The Commentary by LeaderWilliams and Albon makes disheartening reading. Sadly, I believe that the prospects may be even gloomier than they suggest.

The authors claim a linear relationship between money spent per square kilometre of habitat and the health of the populations of black rhinos and elephants in Africa. But there is an alternative interpretation of the data presented in their Fig. 4, revolving about the point representing Kenya. My explanation assumes that the Kenyan datum is reliable, an assumption that rests on the fact that the Kenyan national park system is one of the best-run in Africa.

The relationship portrayed is probably not one of linear increase, but is instead 'flat' through levels of spending represented by Kenya (say, less than $\$ 200$ per $\mathrm{km}^{2}$ ) and only begins to show returns when spending levels increase well into the $\$ 200-300$ per $\mathrm{km}^{2}$ range. This is to say that considerable sums must be spent before returns can be expected, and at lower levels there is no reason to expect even minor success. The authors' citation of the relationship between spending and elephant populations (in the caption to Fig. 4) provides a substantially smaller $r^{2}$ value, indicating even less clarity in that relationship.

I see no conflict between this alternative explanation and the data represented in Fig. 3, which show a linear relationship between effort - as $\log _{\mathrm{c}}$ (total patrol days/ size of area/frequency of rhino sightings in 1980) — and the instantaneous rate of change in black rhino populations in the Luangwa Valley. Restricting analysis to a single area offers an important control in a continent as politically and socially diverse as Africa. The continental relationship may likewise be linear, but the data do not yet support that interpretation to the exclusion of others.

The expectation of disappointment at lower spending levels would take some of the bite out of the frustration, and perhaps fuel the argument for greater expenditure on conservation. We are all in for a long haul, and it is high time that both poor and especially rich nations realized that the fight to save African wildlife will continue to be difficult, frustrating and costly. RANDALL BREITWISCH

\section{Department of Biology,}

University of Dayton,

300 College Park,

Dayton, Ohio 45469, USA

LEADER-Williams REPLIES-The dehorning of rhinos has been discussed by conservationists since the $1950 \mathrm{~s}$, but has not yet been attempted as a means of preventing poaching. This would require considerable resources, because wild rhinos first have to be caught. The Zimbabwe capture unit, for example, is working to capacity in the Zambezi Valley to remove black rhinos to safer parts of the country and catches around 75 animals per year. Nor has it been established whether dehorning could be permanent, for rhinos which lose a whole horn regrow a new one within 3 years.

Dehorning may also be counterproductive. In the short term, a poacher sighting a rhino in thick bush could shoot before finding his quarry is hornless. In the long term, mother rhinos may be less able to protect their calves from predators, such as lions or hyaenas, thereby reducing recruitment. But given the desperate plight of black rhinos, it may now be appropriate to develop a permanent method of dehorning and at least to monitor the survival of one experimental group of dehorned and control rhinos.

On the basis of one outlying point, Breitwisch argues that the relationship between spending and success at protecting rhinos in different countries may suggest a threshold. In fact, a semilog plot does not provide any better fit to the data for rhinos $\left(r^{2}=0.64\right)$ than the simple regression in our Fig. 4. Furthermore, the regression for elephants (not displayed in our article) had outlying points on both lower and upper sides of the line, as expected in such a politically and socially diverse continent as Africa.

More importantly, there is little disagreement in terms of policy. If resources are scarce, we suggested that they must be concentrated at appropriate levels within small areas to achieve given objectives, in this case to prevent a decline of rhinos. The donation to Zambia, which resulted in the linear relationships for Luangwa Valley in our Fig. 3, achieved little because it was insufficiently concentrated and rhinos declined in all areas at rates far in excess of recruitment. Hence, we did not argue for 'dilute' spending because funds used in this way are, ultimately, wasted. Whether the appropriate sum per unit area is derived from an intercept or from Breitwisch's proposed threshold appears to be immaterial.

We also noted that governments in developing countries and charitable conservation agencies do not have sufficient funds to maintain large conservation areas. It may be less disheartening in future if bilateral aid organizations become increasingly involved in conservation for development. At present, only a small fraction of their annual budgets of around $\$ 40,000$ million are spent on conservation. Even a further 5-10 per cent of their budgets, harnessed into well-directed schemes requested by host governments, would greatly help to stem the erosion of the natural resource base of developing countries.

Large Animal Research Group,

N. LEADER-WILLIAMS

Department of Zoology,

34A Storey's Way,

Cambridge CB3 ODT, UK

\title{
A new planet around SN1987A
}

SIR-The discovery in SN1987A of a 0.508 -ms pulsar, with a sinusoidal $3 \times$ $10^{-3} \mathrm{~Hz}$ (peak-to-peak) modulation of 8hour period, has been reported'. The origin of this modulation is intriguing.

A real modulation in the rotation period of the pulsar by pulsations with an 8-hour period can be excluded. The modes of pulsation of degenerate white dwarfs are relatively well understood ${ }^{2}$. The upper limit to the period of the longperiod gravity modes is calculated to be around 1,000 seconds for white dwarfs, in agreement with the observations. For a fixed mass the maximum period is directly proportional to the radius of the star. A neutron star has about the same mass as a white dwarf but is three orders of magni- tude smaller, so the maximum pulsation period will be around 1 second.

A natural explanation for the modulation is that it is due to a body orbiting the neutron star. The semi-major axis of the orbit, around a neutron star of one solar mass $\left(M_{\odot}\right)$, of an object with an 8-hour period is 2 solar radii. This implies a mean orbital velocity of such an object of about $300 \mathrm{~km} \mathrm{~s}^{-1}$. The velocity of the pulsar of $0.23 \mathrm{~km} \mathrm{~s}^{-1}$, deduced from the Doppler shift, is only a lower limit to the true velocity, because the inclination is unknown. This gives a lower limit to the mass of the orbiting planet of $7.7 \times 10^{-4} M_{\odot}$; the most likely mass is about twice this (that is, about the mass of Jupiter).

The planet cannot have existed before 
the supernova explosion because its present orbit would then have been inside the blue giant progenitor star. Even a planet orbiting just above the surface of the star would have been expelled from the system by the explosion because this abruptly reduced the central mass by a factor of 20 . The planet must therefore be composed of matter that came from deep inside the star and went into orbit after the supernova explosion.

When SN1987A exploded, about $18 M_{\circ}$ of matter on the outside was expelled, but the matter in the interior collapsed inwards to form the neutron star. $\mathrm{A} \mathrm{model}^{3}$ suggests that the radial velocity of matter fell below $500 \mathrm{~km} \mathrm{~s}^{-1}$ close to the centre of the envelope. It is conceivable that $1.5 \times$ $10^{-3} M_{\odot}$ of this inner envelope may have gone into orbit around the central core assisted by rotation of the core and a slight departure from spherical symmetry in the explosion.

The matter deep inside SN1987A will consist almost entirely of heavy metals which, within a year of the outburst, will have condensed into grains. Computer simulations of the formation of planets in our Solar System from coalescence of grains ${ }^{4}$ show that the process is very rapid, taking only $100-1,000$ orbital periods. For the putative SN1987A planet this corresponds to less than a year. The simulations also show that the most likely result of such coalescence (especially for more eccentric initial orbits of the dust cloud) is a single large planet, as may be the case around SN1987A.

\section{MARTIN GASKELL}

\section{Astronomy Department,}

Dennison Building,

University of Michigan.

Ann Arbor,

Michigan 48109-1090, USA

1. Middledicth, J. et al. IAU Circ. No. 4735 (1989).

2. Hansen, C. J., Winget, D. E. \& Kawaler, S. D. Astrophys. J. 297, 544-547 (1985)

3. Woosley. S. E. in Supernova $1987 \mathrm{~A}$ in the Large Magellanic Cloud (eds Kafatos, M. \& Michalitsianos, A.) 289-299 (Cambridge Univ. Press, 1988).

4. Isaacman, R. \& Sagan, C. Icarus 31, 510-533 (1977)

suspended object is not "usually done by cementing a mirror on the surface and setting up an interferometer". This technique has been tested without much success, but the transducers used in all the antennas that have been operating and taking data have nothing to do with interferometers.

Finally, the uncertainty principle and the corresponding quantum noise limit the performances of all measuring devices, not only of some of them, and the frequency spectrum of a bar detector is not "a complicated function of its geometry": the longitudinal mode, which is what matters because it is best coupled to the gravitational radiation field, is separated from the other resonances by several hundred hertz.

Most probably, both types of detectors, bars and interferometers, will be pushed to the sensitivity necessary for providing useful astrophysical data. Only their use in observational work will allow an adequate comparison, because computations on paper can be misleading. We tend to believe that both will find favour, but for different classes of events.

\section{G. PALOTTINO G. PIZZELLA}

Dipartimento di Fisica,

Università degli Studi di Roma

"La Sapienza", I-00185 Roma, Italia

their effort to laser interferometer detectors. This is incorrect. Four US groups and others in Italy, Australia and China are actively engaged in research to operate and develop improved resonant bar gravitational-wave detectors.

Resonant bars remain the most sensitive gravitational-wave detectors yet developed and are the only instruments so far used to mount a serious search for gravitational radiation. There has been tremendous development and refinement of the basic design used by Joe Weber two decades ago and the potential remains for vast improvements.

Maddox's statement that resonant detectors are "out of fashion" for mechanical reasons is also completely off the mark. Although larger detectors would give proportionally larger signals, the potential sensitivity of a particular detector also depends on the methods available for motion detection. Maddox states that interferometry is the preferred method for detecting the vibrations of a bar detector but electromechanical transducer schemes, which function by the modulation of an impedance in a superconducting circuit, have been found to be superior. There is no reason to attempt to construct larger bar detectors until the full potential of existing bars has been exhausted by pushing the transducer sensitivity to the maximum. Thus the argument that the larger bars which are needed will be difficult to suspend in a vibration-free manner is specious.

Nor is the issue of the quantum limt of bar detectors an immediate concern. There is a factor of about 1,000 to go from level of $3 \times 10^{-18}$ to the 'quantum limit'. It should even be possible to surpass this limit by using a specially designed transducer to force the bar into a 'squeezed state', analogous to an optical squeezed state, and integrating the existing technologies for low acoustic loss materials and low electrical loss superconducting circuits. In this way, a sensitivity level of $10^{-22}$ could be reached with bar detectors. MARK F. BOCKO Department of Electrical Engineering, University of Rochester,

Rochester, New York 14627, USA

SIR-We fully share the concern (Nature 336, 513; 1988) that the US National Science Foundation may turn down the proposal for a new gravity-wave detector based on laser interferometer and we are equally concerned about the financing of the equivalent European projects. We are convinced, however, that resonant bar detectors have a much greater future than is suggested, and wish to correct some statements about them.

First, bar detectors are not, of course, "suspended so as to pick up mechanical movements from the surroundings" but are substantially isolated by stacks of mechanical filters. In present detectors the external container can be hit with no effect on the output data, so it is difficult to believe that "passing traffic is a nuisance". In an interferometer at least three mirrors are suspended and protected by mechanical filters.

Second, the measurement of the displacement of the free surface of the

\section{Fear of flying}

SIR-Robert Parker (Nature 336, 719; 1988) purports to demonstrate that a Boeing 747 crash could put 250,000 people at risk from the combustion of the depleted uranium counterweight in the tail.

His figures show that on the worst-case assumptions, $8.5 \mathrm{~kg}$ of uranium would reach the atmosphere in respirable form, and that if 250,000 people inhale exactly equal shares of a total of $8 \mathrm{~kg}$ which then concentrates on one kidney in each individual, that kidney reaches its maximum permissible uranium oxide concentration.

May one enquire how all the contaminated air gets uniformly inhaled, why the absorbed material seeks out one kidney, and why the maximum permissible concentration puts people at risk?

D. E. J. THORNTON

9 Burford Lane,

Lymm, Cheshire WA13 OSE, UK

\section{Scientific Correspondence}

Scientific Correspondence is intended to provide a forum in which readers may raise points of a scientific character. They need not arise out of anything published in Nature. In any case, priority will be given to letters of less than 500 words and five references. 